Q. $\mathbf{S}_{\text {cripta }} \mathbf{R}$ vol. 2 (2015): 7-16

\title{
Hacia una literatura sin ficción. Juan José Millás, Javier Cercas y Antonio Muñoz Molina
}

\author{
Towards a non-fiction literature. Juan Jose Millas, Javier Cercas and \\ Antonio Munoz Molina
}

Agnieszka Kłosińska-Nachin

Universidad de Łódź, Polonia

\begin{abstract}
Resumen: El artículo se propone reflexionar en torno a la llamada literatura sin ficción a partir de opiniones (especialmente las expresadas en la prensa diaria) de Juan José Millás (1946), Javier Cercas (1962) y Antonio Muñoz Molina (1956). La predilección por este tipo de literatura, así como una intensa actividad periodística de estos autores, dan lugar a una reflexión más general sobre el papel de la literatura y del escritor en la actualidad, frente a los cambios poco favorables a las humanidades.

Palabras clave: literatura sin ficción, Javier Cercas, Juan José Millás, Antonio Muñoz Molina.
\end{abstract}

\begin{abstract}
This article aims to analyse so-called non-fiction on the basis of opinions (particularly those voiced in the daily press) of Juan José Millás (1946), Javier Cercas (1962) and Antonio Muñoz Molina (1956). The preference for this type of literature and the intense journalistic activity of these authors give rise to more general reflection about the role of literature and the writer today in the face of unfavourable changes in the humanities.
\end{abstract}

Keywords: non-fiction, literature, Javier Cercas, Juan José Millás, Antonio Muñoz Molina.

El papel que ocupa la literatura en la sociedad de hoy se ha vuelto incierto. Por doquiera se mire, nos llegan los mensajes sobre la inutilidad de las humanidades, que no saben hacerse partícipes del vertiginoso progreso de las nuevas tecnologías ni tampoco cooperar con las empresas en sus nuevos proyectos, concebidos a fin de mejorar nuestro día a día. La crisis ha desenmascarado lo que quizás sea el pecado mayor de la literatura: su incapacidad para remediar la incompetencia de los banqueros y de los economistas. Por otro lado, las voces que vienen de los profesionales de las humanidades llevan décadas anunciando cambios trascendentales. Desde que se ha proclamado la muerte del autor (Barthes, 1987), los lectores pasaron a convertirse en autores y la cultura -nos dicen- se ha vuelto más participativa, ya no hay élites ni autoridades, y el público, desde múltiples canales puede contar sus historias, compartirlas y hacérnoslas llegar hasta las pantallas de nuestros móviles para que las disfrutemos en el momento menos esperado del día y de la noche (Jenkins, 2008). Todo ello sellado por los estudios culturales desde los que se nos dice que el texto literario ha perdido el estatus privilegiado que llevaba siglos detentando dentro de la cultura. La pregunta que yo quiero plantear dentro de este panorama (que en absoluto quiero que aparezca 
como apocalíptico) se refiere a los propios autores: ¿Qué papel se asignan ellos mismos? ¿ Cómo reflexionan en torno a su propia actividad? $\dot{\imath} Q u e ́$ rumbos quieren tomar frente a estos cambios tan poco favorables a la literatura, por lo menos la entendida según pautas tradicionales, como texto generado por un autor?

El primer impulso que me llevó a formular estos interrogantes tiene que ver con una conferencia de Antonio Muñoz Molina, pronunciada en la Universidad Complutense de Madrid, en 2008. El conferenciante, antes de entrar en el tema de los límites de la ficción, empezó por recordar a un hombre anónimo, asesinado aquel mismo día (Muñoz Molina, 2008). Este emocionante homenaje de un escritor que, antes de hablar de la literatura, cree conveniente mencionar un acontecimiento de la realidad inmediata me pareció revelador de algo por otra parte evidente: la necesidad de ir apoyándose por parte de los escritores en la realidad y la consiguiente desconfianza ante la estética y la ficción que observamos en la actualidad (Chillón, 2014: 262). Mi intención no es en ningún caso generalizar sino llamar la atención sobre una tendencia que he decidido llamar "hacia la literatura sin ficción" y que se manifiesta, en primer lugar, a través de las relaciones cada vez más estrechas entre la literatura y el periodismo'.

Se me podría objetar fácilmente que el acercamiento al periodismo no significa un alejamiento de la ficción. De hecho, hoy sería difícil mantener la distinción entre la literatura y el periodismo según el criterio de la verdad, dado que, como recuerda un experto en esta materia, no existe la realidad fuera de la dicción (Chillón, 2014: 39-47). Las palabras no reproducen los hechos sino que los representan, acudiendo a un repertorio de recursos que son los mismos para la literatura y el periodismo. Los periodistas se muestran muy conscientes de su capacidad de moldear lo real. Baste recordar la célebre frase de William R. Hearst, quien en 1897 replicó a un corresponsal de Cuba que le decía que tenía que volver a los Estados Unidos, dado que en Cuba no había guerra: "Usted mándeme textos y fotografía, que yo le pondré la guerra" (citado en Suárez, 2008: 50). Otro caso célebre de "un periodismo de ficción" (Suárez, 2008: 47-51) es la ocurrencia de un periodista rumano de publicar un artículo sobre el nacimiento de un niño llamado Yahoo, nombre con el que sus padres hubieran querido celebrar su conocimiento a través de Internet. La noticia dio la vuelta al mundo, con el certificado de nacimiento del niño adjunto. Pero resulta que fue pura invención.

Si es cierto que la ficción inspira a los periodistas (y no sólo me refiero a la invención de lo contado sino sobre todo al abanico de recursos lingüísticos que el periodismo y la literatura comparten), no resulta menos cierto que la realidad hechiza a los escritores. En 1954 Max Aub publicó una biografía titulada Jusep Torres Campalans que recrea la vida del pintor catalán, reproduciendo sus pinturas y fotografías en las que figura junto con Picasso. Y resulta que Aub se lo inventó todo. Pero su invención, igual que el delirio de don Quijote, tiene un impacto en la propia realidad, porque se llagaron a organizar exposiciones del pintor ficticio, como la

\footnotetext{
1 A este respecto, sería interesante indagar las relaciones entre el llamado new journalism y el periodismo y las letras españolas. Según Chillón, si bien las características de dicha tendencia se dan en España a partir de finales de los años sesenta, "el nuevo periodismo hispano ha bebido, sobre todo, en fuentes propias" (2013: 428). En cualquier caso, las relaciones entre ambas áreas han sido promiscuas desde el nacimiento del periodismo, que Chillón relaciona con la aparición de la novela moderna (2013: 155-183).
} 
inaugurada en el Museo Reina Sofía en 2003 en presencia del presidente Aznar. ¿Dónde está el hechizo? ¿Qué aliciente tiene la realidad para un novelista? La respuesta la buscaré en las reflexiones en torno a la lengua y a la expresión literaria de tres autores contemporáneos, Juan José Millás (1946), Javier Cercas (1962) y Antonio Muñoz Molina (1956), los tres galardonados con premios de literatura y periodismo.

En contra del muy alarmante diagnóstico que he formulado al principio, los escritores españoles de hoy se han hecho y se están haciendo muy visibles, en primer lugar gracias a su cooperación con la prensa diaria. Aquí cabe indicar el impresionante auge del columnismo que se registra en España desde la década de los noventa del siglo pasado hasta hoy, como una de las consecuencias de la transición y de la afluencia de los escritores a los periódicos. Así nació la columna personal que no tiene que ceñirse a la actualidad más inmediata y que, según una definición muy citada de Martínez Albertos, corresponde a:
unos guetos privilegiados del periodismo impreso delimitados por los siguientes rasgos: son espacios concedidos como cheques en blanco a escritores de indudable nombradía para que escriban lo que quieran y como quieran, con la condición de que no se extralimiten en el número de palabras previamente acordado y de que respalden con su firma las genialidades o tonterías que decidan exponer en cada uno de sus artículos (Martínez Albertos, 1997: 195).

No pienso adentrarme en la discusión académica acerca de si la columna personal pertenece a la literatura o al periodismo. Más reveladora al respecto me parece la opinión del columnista del País, Juan José Millás, que en una entrevista digital con sus lectores dice lo que sigue:

Yo desde luego sí tengo estos dos géneros [la literatura y el periodismo] completamente confundidos. No sé dónde empieza el periodismo y termina la literatura. Para mí el periódico es un artefacto literario. Otra cosa es que sea un buen o un mal artefacto. Cuando escribo periodismo, pues, no tengo la impresión de no estar haciendo literatura. Escribo mis columnas y mis novelas desde un mismo sitio (Millás, 2001).

Javier Cercas, otro escritor periodista del que pienso ocuparme en estas páginas, afirma:

Yo llegué al periodismo muy tarde y para mí es un deslumbramiento, un gran descubrimiento, y he intentado aprovecharme de cosas del periodismo en los libros que he escrito (citado en Martín Rodrigo, 2015).

Antonio Muñoz Molina igualmente se siente en deuda con el periodismo:

Una gran parte de los relatos y de los artículos que yo he escrito, y entre ellos algunos de los que puedan parecer más fantásticos y extravagantes, proceden de noticias del periódico (Muñoz Molina, 1993: 19).

Para apreciar el peso de este tipo de afirmaciones, cabría cotejar la relación entusiasta de los escritores de hoy ante el periodismo con la actitud más escéptica que tenían los modernistas frente a su también intensa actividad periodística. Recordemos aquellas frases del "Prefacio" a Cantos de vida y esperanza de Rubén Darío: "Yo no soy un poeta para muchedumbres. Pero sé que indefectiblemente 
tengo que ir a ellas" (2006: 45). Y estas opiniones del cubano Julián del Casal, de 1893:

El periodismo puede ser, dado el odio que en él se respira hacia la literatura, la mano benefactora que, llevando el oro a nuestros bolsillos, coloque el pan en nuestra mesa y el vino en nuestro vaso. ¡Ay!, pero no será nunca el genio tutelar que nos ciñe la corona de laurel (Casal, 1963: 271).

Y más adelante:

Escribiendo con frecuencia, como lo hace el periodista, la pluma adquiere cierta soltura, pero a cambio de esto, ¡cómo se aprende a cortejar la opinión pública, cómo a aniquilar las ideas propias, cómo a descuidar el pulimento de la frase, cómo a expresar lo primero que se ocurra y cómo a aceptar el gusto de los demás!" (Casal, 1963: 271).

El periodismo para muchos de los autores de principios del siglo pasado fue un mal que, por razones económicas, tenían que aceptar, pero la dedicación a él alejaba de la composición de la anhelada obra de arte total, capaz de abarcar el Absoluto. Frente a ello, el espectacular desarrollo de la crónica modernista, género periodístico con un tratamiento subjetivo y artístico de los acontecimientos inmediatos, es un paso de gigante hacia una concepción híbrida de la literatura a la que hoy tachamos de postmoderna o, siguiendo a Steiner, le atribuimos el calificativo de postficción (Chillón, 2014: 40). En cualquier caso, la diferencia en la valoración del periodismo nos permite apreciar el giro esencial que se ha operado a lo largo del último siglo en la consideración de la actividad literaria, especialmente en su posicionamiento frente a la realidad más inmediata. Aunque la tendencia a los hechos no es nada nuevo en la literatura (recuérdese que los realistas y los naturalistas tenían que documentarse sobre el mundo antes de ponerse a escribir), el intensificado apetito de realidad es inseparable de la cultura contemporánea, irrumpiendo en todas sus manifestaciones. Fuera de las evidentes motivaciones económicas que empujan, igual que hace un siglo, a los autores de hoy a escribir para los periódicos (Marín Malavé, 2011: 54-59), detrás de su actividad hay que ver el deseo de mantenerse en permanente contacto con su público, hecho que sobresale todavía más si tenemos en cuenta el carácter periódico del columnismo actual. Así, el escritor y su producción se convierten en elementos de la comunicación social, el texto se democratiza, y la autoría ya no se concibe como una actividad propia de un demiurgo (Hopfinger, 2011: 13-23), por lo menos desde la parcela del campo literario que estoy examinando.

Veamos, pues, cómo los autores columnistas actuales conceptualizan el papel de la literatura y el del autor. En primer lugar, es interesante y hasta aporta consuelo observar que, al democratizarse el acceso al texto y al autor (téngase en cuenta que se realizan entrevistas digitales en las que los autores contestan a las preguntas formuladas por los internautas), este último no renuncia al privilegio de ejercer su autoridad en materia de la lengua y no duda en arremeter en contra de los que no saben aplicar debidamente las normas. Para poner un ejemplo, en dichas entrevistas, Juan José Millás suele corregir a los internautas poco hábiles en ortografía. El mismo autor, en una columna titulada "La coma" de 2005, se refiere al lema publicitario del Banco de Santander: "Gracias Isabel por hacernos el mejor del mundo" y comenta: "No sabemos qué controles ha pasado esta campaña, pero 
está mal escrita. El texto debería decir: "Gracias, Isabel, por hacernos el mejor del mundo". Y recuerda la regla: "Isabel va entre comas porque se trata de un vocativo" (Millás, 2005)². En 2007, en cambio, se mete con la Comunidad de Madrid que en su campaña para promover la lectura utilizó un anuncio poco afortunado con una chica joven que decía: "No puedo vivir sin salir por ahí, mis taconazos y los poetas malditos". A Juan José Millás le irrita la ocurrencia de privilegiar a los poetas malditos "que además son, por lo general, franceses" (Millás, 2007), en una campaña financiada con fondos públicos y se pone sarcástico:

¿Qué habría impedido decir no puedo vivir sin salir por ahí, mis taconazos y José María Pereda? Quien dice José María Pereda dice Marcelino Menéndez Pelayo o Benito Pérez Galdós. No puedo vivir sin salir por ahí, mis taconazos y Benito Pérez Galdós (Millás, 2007).

Para resaltar el carácter absurdo de una campaña que debería incitar a leer, se lanza a proponer sus propios lemas basados en la misma ocurrencia que el oficial: "No puedo vivir sin mi Almax para el ardor de estómago y Unamuno" (Millás, 2007). Así que el escritor se erige desde las páginas diarias de un periódico en guardián de la lengua y la democratización del medio le sirve para ejercer su autoridad con más eficacia y darle mayor alcance a su mensaje.

Curiosamente, tanto Juan José Millás como Javier Cercas reflexionan en torno a lo que es la literatura a partir de textos no literarios. Juan José Millás comenta una nota hallada en el bolsillo del cadáver de un oficial del submarino ruso Kursk. En agosto de 2000 el mundo estaba sacudido por la noticia sobre el hundimiento del navío a raíz de unas explosiones. Las autoridades rusas dieron a conocer la noticia varios días después de lo ocurrido, de modo que los intentos de rescate empezaron con mucho retraso, cuando todos los tripulantes habían fallecido. La nota comentada por Millás reza lo siguiente:

13.15. Todos los tripulantes de los compartimientos sexto, séptimo y octavo pasaron al noveno. Hay 23 personas aquí. Tomamos esta decisión como consecuencia del accidente. Ninguno de nosotros puede subir a la superficie. Escribo a ciegas (citado en Millás, 2000).

Para Juan José Millás es una obra maestra. Y añade: "En situaciones extremas, la literatura sale a presión, como por la grieta de una tubería reventada" (Millás, 2000). De modo que el relato nace en la realidad misma, en un impulso innato que empuja a contar cosas que no requieren la intervención del talento de un artista. Al mismo tiempo, Millás confía en el lector y en su aptitud para reconstruir la historia del Kursk, un lector que se hace responsable de lo contado en la misma medida que lo es el propio autor. Por otro lado, el columnista relaciona el acto de escribir con la situación del oficial: el pánico extremo y la inseguridad acerca de si alguien leerá lo escrito.

\footnotetext{
2 Aunque existen varias ediciones de los artículos que se comentan a continuación, cito de los archivos de los periódicos en los que aparecieron originariamente. Dos razones me han empujado a optar por esta solución: primero, por razones que desconozco, en las ediciones de los articuentos de Millás no se suele proporcionar la fecha ni la fuente de los textos (véase a este respecto la edición de Seix Barral de 2011 ). En segundo lugar, gracias a la dirección de la página web, el lector tiene un acceso facilitado a los textos comentados.
} 
En la conferencia citada sobre los límites de la ficción, Antonio Muñoz Molina asegura que hay historias reales que no admiten que se las remiende con la ficción porque olerían a mentira. La nota del Kursk parece ser una de estas historias. La extrema concisión del texto que roza la exactitud matemática (fijense en la cantidad de números que aparecen en una nota tan breve) genera una intensa carga emocional, todo ello por la inminencia de la muerte que es lo ausente y lo presente a la vez.

Llamativamente, Javier Cercas, otro escritor que reflexiona sobre la literatura en la prensa, se deja hechizar por un texto cuyo origen recuerda el de la nota del Kursk. En enero de 2015 el autor de Los Soldados de Salamina publica en El País Semanal un artículo titulado "Literatura más allá de la literatura" que empieza del siguiente modo: "Uno de los mejores textos literarios que he leído en mi vida no es un texto literario" (Cercas, 2015). Más adelante nos enteramos de que el texto que tanto entusiasmo ha inspirado a Javier Cercas es un discurso del escritor israelí David Grossman, leído por él en el funeral de su hijo Uri, muerto en un combate en Líbano donde su carro fue alcanzado por un misil antitanque de Hezbolá. Al ver que los periodistas presentes en el entierro reproducían el texto, Grossman lo corrigió y lo mandó a los periódicos. En España el discurso de Grossman fue publicado por El País. Para Cercas, es un texto sobrecogedor e "infinitamente más literario que infinidad de textos literarios" (Cercas, 2015). ¿Qué es lo que le confiere el carácter literario? Para contestar esta pregunta, Cercas se apoya en otra carta que un padre le dirige a su hijo, cuando éste se encuentra en Oxford, con una beca, dispuesto a hacerse escritor. El hijo no es otro que Naipaul y su padre le escribía así:

\begin{abstract}
¿A qué crees que se reduce la literatura? A escribir con las tripas, no con la cabeza. La mayoría escribe con la cabeza. Si el delincuente semianalfabeto escribe normalmente una larga carta a su novia, será como la mayoría de las cartas de semejantes personas. Si el delincuente escribe la carta justo antes de ser ejecutado, será literatura (citado en Cercas, 2015).
\end{abstract}

Y luego viene el comentario de Cercas:

Esa es la respuesta. La literatura es lo que se escribe como si uno estuviera a punto de ser ejecutado; o, mejor aún, como si ya hubiese sido ejecutado, que es como escribe su texto Grossman. La gran literatura es precisamente eso: lo que está justo en el borde de la literatura. O un poco más allá (Cercas, 2015).

El oficial del Kursk escribía cuando ya era ejecutado; de hecho, cuando el contenido de su nota estremecedora se reveló al primer lector, ya estaba muerto. Del mismo modo, Grossman escribe desde lo irreversible, sobrecogido por un pánico que sólo la muerte puede infundir. La palabra no aporta consuelo, pero se impone, como dice Millás, "para contarlo" (Millás, 2000). Según Cercas, estamos ante una literatura más allá de la literatura, porque no coincide con las expectativas literarias del momento. Es una literatura que deja de inventar porque en la realidad se agazapan relatos mucho más insólitos y estremecedores que los que un autor podría inventar instalado cómodamente en su despacho. Y aquí tocamos una característica esencial de esta literatura que renuncia deliberadamente a la ficción: su compromiso con lo real. Sería imperdonable en este contexto no recordar la frase 
de Javier Cercas de El impostor, una novela de 2014: "Literatura sirve para salvar a un hombre, si es de adorno, a la mierda la literatura" (Cercas, 2014).

La mención del último libro de Javier Cercas no nos hace alejarnos mucho del marco del periodismo. La novela, género que Cervantes llamó "escritura desatada", acoge la no ficción desde por lo menos A sangre fría (1966) de Truman Capote. En las letras hispánicas es indispensable mencionar Relato de un náufrago (1970) y Crónica de una muerte anunciada (1981), ambos precedidos por artículos en prensa que García Márquez dedicó a los asuntos tratados antes de darles la forma de libros. Recordemos que sobre la Crónica dirá varios años más tarde "La mejor fórmula literaria es siempre la verdad" (citado en García de León, 1998: 343). En la actualidad, una narración sin ficción resulta extremadamente fecunda desde lo que se ha llegado a denominar postmemoria (Potok, 2012: 10). Dicho concepto ha sido desarrollado en relación con Holocausto por Marianne Hirsch (1997: 22) y remite a relatos de aquellos autores que, al no tener recuerdos directos de los acontecimientos traumáticos, acuden a la memoria ajena de los que sí todavía se acuerdan. En el caso de la narrativa española, lo traumático se concentra en torno a la guerra civil y la reciente proliferación de relatos en torno a ella se debe indudablemente a que pronto desaparecerán los que todavía conservan recuerdos directos de aquella contienda ${ }^{3}$. Otro concepto que se maneja para explicar la necesidad de recurrir a la literatura a fin de contar la Historia es el de la metaficción historiográfica (Hutcheon, 1988: 5; Lluch Prats, 2004: 298) que corresponde a un tipo de relato autorreflexivo que se propone compensar las carencias y los huecos de la historiografía oficial, en la que lo humano y lo particular no tienen cabida. Al mismo tiempo, las metaficciones historiográficas ponen al desnudo el inquietante parecido entre la escritura histórica y la ficticia.

En su conferencia sobre los límites de la ficción (2008), Antonio Muñoz Molina cuenta que pertenece a la generación de los nietos de los que participaron en la guerra civil y recuerda el ambiente de misterio en que creció él mismo y sus coetáneos. Era un ambiente en que los relatos estaban en el aire, relatos sin acabar cuyo núcleo tenía que ver con la guerra civil. Llegados a la edad madura y algunos de ellos convertidos en autores, los representantes de esta "generación de nietos" o bien podían retomar estos relatos, rellenando los huecos con la ficción (es el caso de Soldados de Salamina) o bien convertir la literatura en una herramienta de investigación, como en el caso de El impostor.

En esta última novela, Javier Cercas cuenta la historia de Enric Marco, un hombre que se hizo pasar por un superviviente del campo de concentración de Flossenbürg y llegó a presidir la Amical de Mauthausen, una asociación que agrupa a los deportados del nazismo y a sus familiares. De su conducta heroica, de los sufrimientos en el campo de concentración y de su resistencia ante el franquismo habló en múltiples conferencias y charlas que impartió en los colegios y en las universidades, hasta en el parlamento español, conmoviendo a los diputados hasta arrancarles lágrimas. En 2005 Benito Bermejo Sánchez, un modesto historiador, lo desenmascaró justo antes de que interviniera en la celebración de las conmemoraciones de Mauthausen. Ante una impostura tan inusitada, Javier Cecas

\footnotetext{
3 Para apreciar la proliferación de la narrativa española más actual, consúltese el artículo de Magda Potok (2012).
} 
acudió a diversos archivos, consultó a historiadores, habló con gente que conocía a Marco y, por fin, durante largas horas entrevistó y grabó a su protagonista para desentrañar su enigma y para, al final salvarle, reconciliándole con la realidad a través de su novela sin ficción. ¿̇Lo reconcilió? En una entrevista que acordó a la radio SER, Marco, hablando de sí mismo en tercera persona, afirmó una vez más su misión divulgativa: "Tuve gran entereza siempre. Enric Marco hizo cosas necesarias, porque la historia es muy árida de explicar. Esa credibilidad me permitía introducir la verdad" (Antón, 2014). Javier Cercas, al comentar la entrevista, se negó a "entrar en polémicas con su personaje" (Antón, 2014).

De modo que Cercas no llegó, a través de su relato sin ficción, a salvar a su personaje aunque sí, a dignificarlo, como observa Vargas Llosa en su reseña sobre El impostor (Vargas Llosa, 2014). Tal dignificación se consigue, por ejemplo, mediante una recurrente comparación con don Quijote en la que ambos personajes, una vez llegados a los 50 años, se lanzan a reinventar sus vidas, dotándolas de heroicidad, fantasía y aventuras. Pero la verdad de esta novela sin ficción no radica únicamente en desvelar el mecanismo de las mentiras de un mentiroso. De hecho, como reconoce Cercas en otra parte, Marco es sólo una excusa para hablar de unas verdades mucho más generales: "El tema invisible es otro: Enric Marco es lo que somos... pero a lo bestia" (Martínez, 2014). Inventarse un pasado viable para hacer frente a un futuro nuevo es lo que hicieron muchos de los españoles en los años de la transición ${ }^{4}$. De modo que la verdad y el valor (valor en sentido de importancia y audacia a la vez) de El impostor residen en lanzar un debate en torno a cómo el caso Marco ha sido socialmente posible.

A la luz de lo expuesto, para los tres autores españoles comentados, la fórmula vargasllosiana de "la verdad de las mentiras" se ha quedado corta a la hora de enfrentarse con la complejidad y las incertidumbres de sus propias realidades, que por sí mismas incluyen gran dosis de mentiras. Así que la verdad hay que buscarla en la realidad misma, una realidad que las vicisitudes de la Historia han empañado tanto que ha perdido la transparencia y el brillo que nos gustaría que tuviera. En última instancia, los escritores españoles no han dejado de intervenir en nuestras vidas y, por lo visto, están muy lejos de desaparecer de nuestro horizonte. Todo lo contrario.

\section{Bibliografía}

ANTÓN, J. (2014): "El periodismo, la literatura y el mentiroso". El País, 22/11/2014. Disponible en:

http://cultura.elpais.com/cultura/2014/11/21/actualidad/1416593488 878947.html. Consultado el 1/09/2015.

BARTHES, R. (1987 [1968]): "La muerte del autor". R. Barthes: El susurro del lenguaje. Barcelona: Paidós Ibérica, pp. 65-71.

\footnotetext{
${ }^{4}$ Compárese con una de las afirmaciones del lúcido ensayo de Muñoz Molina Todo lo que era sólido: "La idealización de la segunda república y sobre todo del bando republicano en la guerra se correspondía con una amplia ignorancia sobre los hechos comprobados" (2013: 15).
} 
CASAL, J. del (1963 [1893]): "Bonifacio Byrne". J. del Casal: Prosas. La Habana: Consejo Nacional de Cultura, p. 271.

CERCAS, J. (2014): El impostor. [s/l]: Literatura Random House, edición para Kindle.

- (2015): "Literatura más allá de la literatura". El País, 04/01/2015. Disponible en: http://elpais.com/elpais/2015/01/02/eps/1420212864_912263.html. Consultado el 17/09/2015.

CHILLÓN, A. (2014 [1999]): La palabra facticia. Literatura, periodismo y comunicación. Universitat Autónoma de Barcelona - Universitat Jaume I de Castelló - Universitat Pomeu Fabra - Universitat de Valencia: Aldea Global.

DARÍO, R. (2006 [1905]): "Prefacio". R. Darío: Cantos de vida y esperanza. Salta: Biblioteca de Textos Universitarios.

GARCÍA DE LEÓN, M. E. (2000): "Literatura periodística o periodismo literario". F. SEVILLA ARROYO y C. ALVAR EZQUERRA (coords.): Actas del XIII Congreso de la Asociación Internacional Hispanistas, Madrid, 6-11 de julio de 1998, vol. 4, pp. 335-343.

HIRSCH, M. (1997): Family Frames: Photography and Narrative of Postmemory. Cambridge: Harvard University Press.

HOPFINGER, M. (2011): „Współczesne przemiany literatury a dziennikarstwo”. K. WOLNY-ZMORZYŃSKI, W. FURMAN, J. SNOPEK (red.): Dziennikarstwo a literatura w XX i XXI wieku. Warszawa: Poltext, pp. 13-23.

HUTCHEON, L. (1988): A Poetic of Postmodernism. History, Theory, Fiction. New York London: Routledge.

JENKINS, H. (2008 [2006]): Convergence culture: la cultura de la convergencia de los medios de comunicación. Barcelona: Paidós Ibérica.

LLUCH PRATS, J. (2004): "La dimensión metaficcional en la narrativa de Javier Cercas". L. BLINI y M. V. CALVI (eds.): Escritura y conflicto. Actas del XXII Congreso AISPI, Catania - Ragusa, 16-18 mayo 2014, pp. 293-306.

MARÍN MALAVÉ, M. del R. (2011): El columnismo de Juan José Millás en relación con su narrativa. Análisis de sus columnas en el País (1990-2018). Málaga: Servicio de Publicaciones de la Universidad de Málaga.

MARTíN RODRIGO, I. (2015): "Javier Cercas: "No he visto a nadie más pesimista sobre el periodismo que los propios periodistas"ll. Abc, 16/04/2015. Disponible en: http://www.abc.es/cultura/20150416/abci-javier-cercasromero-murube-201504151739.html. Consultado el 21/09/2015.

MARTínEZ, L. (2014): «Javier Cercas "Enric Marco es lo que somos... pero a lo bestia"ll. El Mundo, 14/11/2014. Disponible en: http://www.elmundo.es/cultura/2014/11/14/54653062e2704e39528b4576.html. Consultado el 29/09/2015.

MARTíNEZ ALBERTOS, J. L. (1997): El ocaso del periodismo. Barcelona: CIMS. 
MILLÁS, J. J. (2000): "Escribir". El País, 03/11/2000. Disponible en: http://elpais.com/diario/2000/11/03/ultima/973206002 850215.html. Consultado el 18/09/2015.

- (2001): "Entrevistas digitales". El País, 02/04/2001. Disponible en: http://cultura.elpais.com/cultura/2001/04/02/actualidad/986233453_986233 535.html. Consultado el 29/08/ 2015.

- (2005): "La coma". El País, 22/07/2005. Disponible en: http://elpais.com/diario/2005/07/22/ultima/1 121983202 850215.html. Consultado el 10/09/2015.

- (2007): "Gillipolleces". El País, 27/04/2007. Disponible en: http://elpais.com/diario/2007/04/27/ultima/1 177624802 850215.html. Consultado el 3/09/2015.

- (2011): Articuentos. Barcelona: Seix Barral.

MUÑOZ MOLINA, A. (1993): La realidad de la ficción. Sevilla: Renacimiento.

- (2008): "Los límites de la ficción". Disponible en: https://www.youtube.com/watch? v=UFR5hfMSsPU. Consultado el 10/09/2015.

- (2013): Todo lo que era sólido. Barcelona: Seix Barral.

POTOK, M. (2012): "Estrategias literarias para la recuperación de la memoria histórica. La narrativa actual frente a la guerra civil". Études Romanes de Brno, 33/2, pp. 9-20.

SUÁREZ, L. S. (2008): "Literatura de no ficción, periodismo de ficción". Cuenta y Razón, julio-agosto, pp. 47-51. 